\title{
Space-charge-sustained microbunch structure in the Los Alamos Proton Storage Ring
}

\author{
S. Cousineau, V. Danilov, and J. Holmes \\ Oak Ridge National Laboratory, Oak Ridge, Tennessee, USA \\ R. Macek \\ Los Alamos National Laboratory, Los Alamos, New Mexico, USA \\ (Received 13 May 2004; published 8 September 2004)
}

\begin{abstract}
We present experimental data from the Los Alamos Proton Storage Ring (PSR) showing long-lived linac microbunch structure during beam storage with no rf bunching. Analysis of the experimental data and particle-in-cell simulations of the experiments indicate that space charge, coupled with energy spread effects, is responsible for the sustained microbunch structure. The simulated longitudinal phase space of the beam reveals a well-defined separatrix in the phase space between linac microbunches, with particles executing unbounded motion outside of the separatrix. We show that the longitudinal phase space of the beam was near steady state during the PSR experiments, such that the separatrix persisted for long periods of time. Our simulations indicate that the steady state is very sensitive to the experimental conditions. Finally, we solve the steady-state problem in an analytic, self-consistent fashion for a set of periodic longitudinal space-charge potentials.
\end{abstract}

DOI: 10.1103/PhysRevSTAB.7.094201

PACS numbers: 29.27.Bd

\section{INTRODUCTION}

In recent years, many theoretical analyses and a handful of experiments have been conducted to explore the existence of steady-state density perturbations in high intensity coasting beams. Theoretical work has focused on modeling solitary wave structures perpetuated by collective effects, such as resistive and reactive wake impedances [1-3], space-charge impedances [4,5], and rf resonator impedances [6]. Some supportive experimental investigations have included measurements of beam transfer functions and of diffusion rates at Fermilab [7]. Additionally, solitons in bunched beams operating above transition have been studied in the RHIC machine [3]. The general consensus in the literature is that solitary structures can and should exist in high intensity beams, both above and below transition.

In a paper by Koscielniak et al. [8], the effect of longlived longitudinal density fluctuations was studied using rf gymnastics to insert large, relatively low-frequency "holes" in the longitudinal phase space of the CERN Proton Synchrotron (PS) beam. The lifetime and dynamics of the holes was observed during a subsequent $\mathrm{rf}$ capture and acceleration. Despite a significant beam energy spread which would predict fast decoherence, the induced structure was sustained for thousands of turns. Through the experimental findings, simulations, and analytic analysis, Koscielniak et al. make the case that space charge is the underlying cause of the sustained structure. According to their arguments, holelike structures should occur ubiquitously in synchrotrons operating below transition, though to date such observations have been limited to the CERN PS Booster, and, to some extent, the Fermilab Antiproton Accumulator.
In this work we present findings of sustained longitudinal structure in the Los Alamos Proton Storage Ring (PSR) high intensity beam. In a 2002 experimental data set acquired for study of an unrelated phenomenon, the 201.25 MHz linac microbunch structure is found to persist in the ring during storage for over a thousand turns, without any rf bunching. Both chopped and unchopped beam data sets for multiple beam intensities clearly show the phenomenon. Additionally, the data show a strong correlation between the strength of the structure and the beam intensity, indicating a space-charge dependence. Simulations of the phenomenon, presented in the following sections, show holelike structures in the longitudinal phase space, similar to those described by Koscielniak et al. These structures are enclosed by a well-defined separatrix, with particles executing unbounded motion outside of the separatrix. In this sense, the system is characterized by a pattern of alternating overdensities and empty phase space buckets, occurring exactly at the linac microbunch frequency, and giving rise to the experimentally observed, long-lived longitudinal structure. The lifetime of the structure is found to be sensitive to the stored beam intensity, the beam energy spread, and the rate of beam injection during accumulation. The steady state occurs only for a finite set of beam conditions, as will be derived in a self-consistent, analytic fashion in Sec. IV.

The idea that space charge contributes to the longevity of the microbunch structure is counterintuitive, since it requires that regions of high particle density are sustained between the empty buckets, despite strong repulsive forces. For common statistical distributions, such as the Boltzmann distribution, the maximum particle density always occurs at the minimum of the potential. The 
opposite is true here: The maximum particle density occurs at the maximum of the potential. A periodic phase space distribution is maintained through a combination of space-charge effects and motion across the phase space due to energy spread in the beam. As particles drift across the phase space, they lose energy and slow down while traversing the increasing sides of the microbunch potentials. For the right balance of space-charge potential and energy deviation, some particles will have just enough energy to overcome the potential barrier. These critical particles will slow down to a near stop at the peak of the microbunch, before arriving on the decreasing side of the potential and regaining the lost energy; thus, these particles lie near the separatrix. The slow motion of the particles near the peak of the potential sustains the high particle density in that region. Likewise, the fast motion of the particles in the wells of the potential sustains the low particle density there. Technically, the system is also capable of trapping particles inside the separatrix, but because of the PSR injection conditions, particles are never injected inside of the empty phase space regions, i.e., there is no longitudinal painting. All particles are injected initially into the high density regions, where they begin their cycle of slow and fast motion across the high and low density regions.

In this paper, we present our experimental findings, along with particle tracking simulations of the experiments, and theoretical modeling of the phenomenon. The paper is organized as follows: In Sec. II we analyze the experimental data, in Sec. III we perform detailed simulations of the experiments, and in Sec. IV we derive steady-state, self-consistent solutions for a set of periodic longitudinal space-charge potentials.

\section{EXPERIMENTAL OBSERVATIONS}

The Los Alamos Neutron Science Center facility accelerates $\mathrm{H}^{-}$ions through a linac to an energy of $800 \mathrm{MeV}$, after which they are strip-foil injected as protons into a $90.26 \mathrm{~m}$ circumference accumulator ring for bunch train compression and extracted to a transfer line for neutron spallation. Under normal operating conditions, the PSR accumulates approximately $3.5 \times 10^{13}$ protons per pulse in about $675 \mu \mathrm{s}$, with a repetition rate of $20 \mathrm{~Hz}$. A singleharmonic, rampable rf cavity is used to maintain the beam gap, and at the end of the accumulation cycle a single-turn beam extraction is performed. These conditions can be altered considerably for beam physics experiments.

The experiments presented here were performed as part of a longitudinal instability threshold study. ${ }^{1}$ Two ferrite inductive inserts used in the PSR to provide passive longitudinal space-charge compensation cause a strong instability in the beam when the inductors are at

\footnotetext{
${ }^{1}$ Data were taken on September 12, 2002.
}

room temperature [9]. The instability is microwavelike in nature and arises from a resonance in the ferrite inductors. The frequency of the ferrite instability is normally around $72 \mathrm{MHz}$, but was closer to $78 \mathrm{MHz}$ in the threshold experiments. In this paper, we focus exclusively on the $201 \mathrm{MHz}$ microbunch structure phenomenon, and filter the data where necessary to eliminate contributions from higher order modes of the ferrite instability. The analysis of the threshold effect for the ferrite instability is presented in a separate work [10].

Two experimental data sets are included in our analysis: One corresponds to chopped beam conditions, where a $200 \mathrm{~ns}$ bunch train occupies the $358 \mathrm{~ns}$ revolution period, and the other corresponds to unchopped beam conditions, where the bunch train occupies the entire $358 \mathrm{~ns}$ period. No rf bunching was applied in either case. For both sets of conditions, the beam was accumulated for $200 \mu \mathrm{s}$ (559 turns), and then stored for an additional $500 \mu \mathrm{s}$. Multiple data sets were taken over a range of beam intensities. The beam intensity in the PSR is varied by either reducing the current in the linac using a set of jaws or by chopping the beam at the source to eliminate alternate pulses; in either case, the accumulation time is unaltered. Turn-by-turn longitudinal profiles are recorded with a wall current monitor at a sampling rate of $2 \mathrm{GHz}$.

The $2.8 \mathrm{MHz}$ beam revolution frequency in the PSR is the 72nd subharmonic of the $201.25 \mathrm{MHz}$ linac microbunch frequency. Therefore, linac microbunches are stacked on top of one another during beam injection into the ring, creating a persistent $201.25 \mathrm{MHz}$ structural component in the longitudinal signal during accumulation. The energy spread in the linac beam is best fit by a biGaussian distribution with $66 \%$ of the beam at $\sigma_{\text {rms }}=$ $0.69 \mathrm{MeV}$, and the balance at $\sigma_{\mathrm{rms}}=0.28 \mathrm{MeV}$ [9]. Because of the large space-charge gradient of the microbunches, along with the sizable energy spread, the microbunch structure of a single injected linac pulse without any $\mathrm{rf}$ bunching dissipates in $\lesssim 30$ turns. Treating the microbunches independently, a similar behavior would be expected in the accumulated beam signal: The $201 \mathrm{MHz}$ signal is expected to disappear within $\sim 30$ turns after the end of injection. However, under the influence of collective effects, and for a certain balance of experimental conditions, we find that the $201 \mathrm{MHz}$ structure can be sustained in the beam for long periods of time, possibly even indefinitely.

Figures 1 and 2 show wall current monitor signals plotted in waterfall form for a $210 \mathrm{nC}$ chopped beam and for a $720 \mathrm{nC}$ unchopped beam. In this format, the time along the bunch train for a single turn is plotted on the horizontal axis, and the turn number is plotted along the vertical axis. The end of beam injection occurs at 559 turns, after which the beam is stored for another 1400 turns. For the chopped beam, the $201 \mathrm{MHz}$ microbunch 


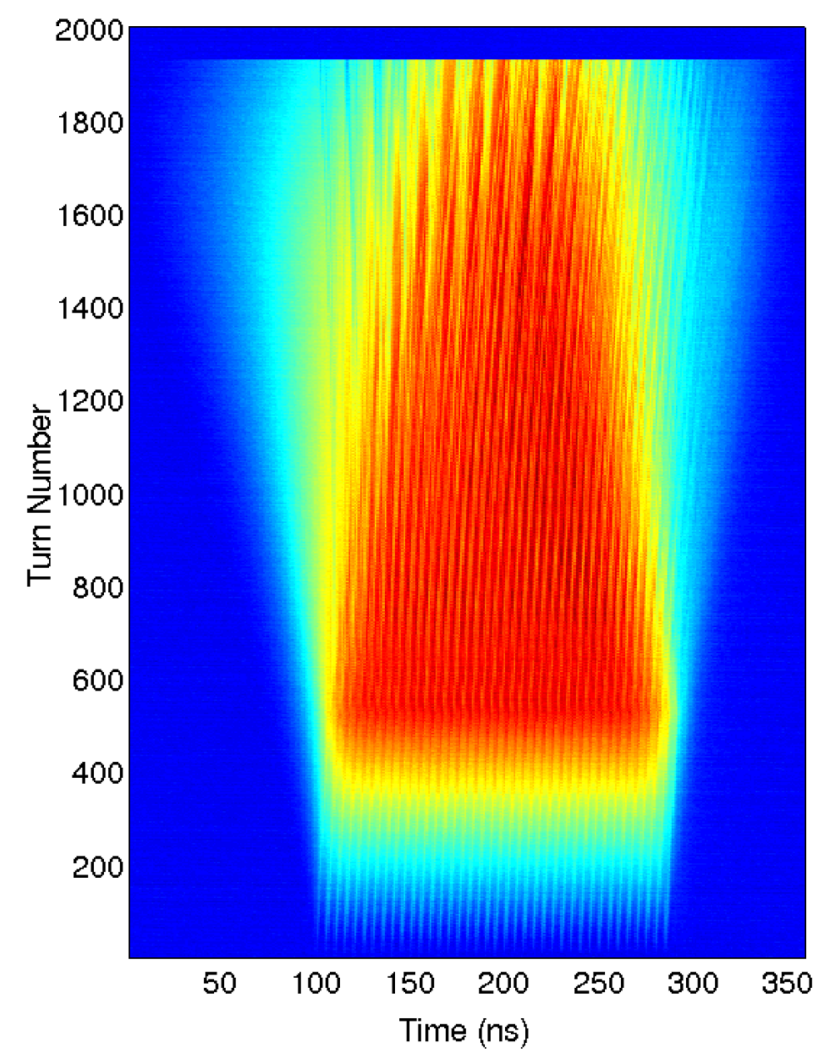

FIG. 1. (Color) Waterfall plot of the wall current monitor signal for the chopped beam experiment. The time in a single pulse is plotted on the horizontal axis, and the turn number is plotted on the vertical axis. Beam injection ends at 559 turns. The high frequency variations in color indicate the microbunch structure of the beam. The beam gap region is displayed in blue.

structure, visible as the high frequency density (color) variations, persists for at least 800 turns after the end of injection. The microbunch structure is even more long lived in the unchopped beam case, remaining strong throughout the entire storage, up until the beam is extracted from the ring at 1950 turns. The longer wavelength (lower frequency) waves seen at the end of the storage in both the chopped and unchopped beam are related to the ferrite instability, which we do not consider here.

A more precise method of characterizing the behavior of the $201 \mathrm{MHz}$ microbunch structure is by monitoring the strength of the corresponding harmonic (harmonic number 72) of the longitudinal beam signal during injection and storage. In order to eliminate possible contributions from higher order harmonics of the ferrite instability, a bandpass filter was applied to the data before the FFTs were taken; the bandpass region was taken from 175 through $210 \mathrm{MHz}$, sufficient to exclude the nearest harmonics of the ferrite instability. Figures 3(a) and 3(b) show the analysis for the chopped and unchopped beam data presented in Figs. 1 and 2, respectively. The results are surprising: For the chopped beam conditions (Fig. 1),

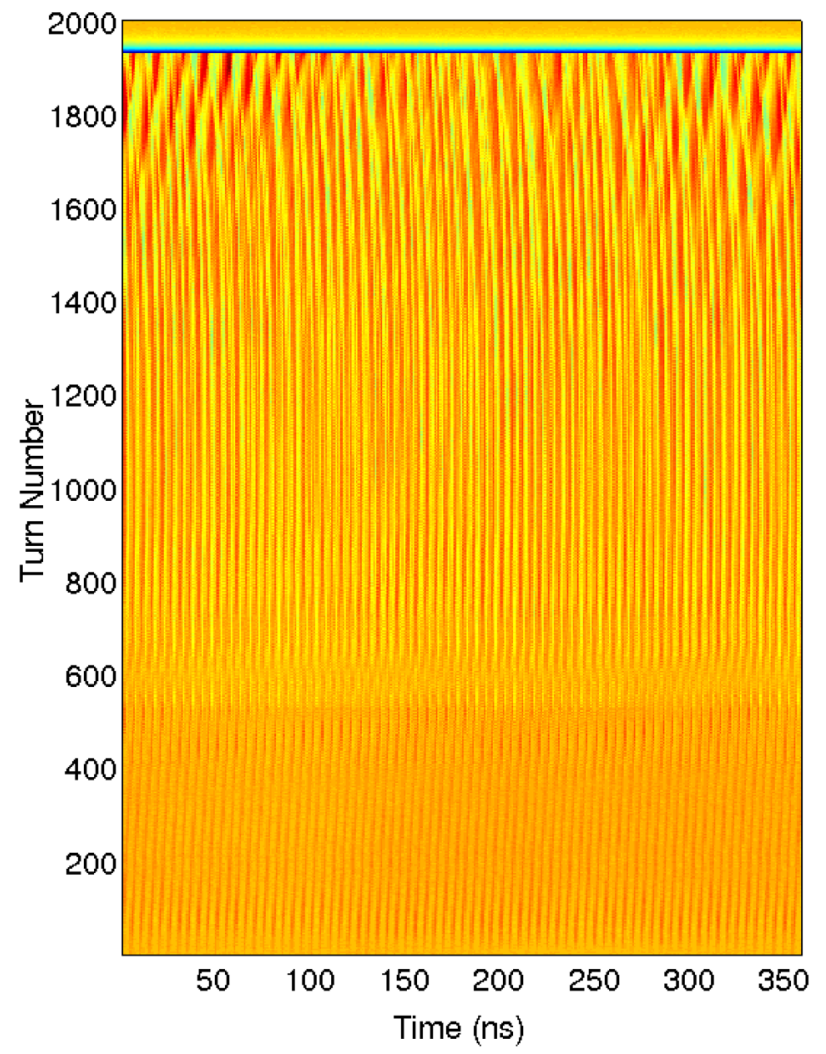

FIG. 2. (Color) Waterfall plot of the wall current monitor signal for the unchopped beam experiment. The time in a single pulse is plotted on the horizontal axis, and the turn number is plotted on the vertical axis. Beam injection ends at 559 turns. The high frequency variations in color indicate the microbunch structure of the beam.

the harmonic is strong throughout the injection period, during which the beam is constantly supplied at the linac frequency. However, instead of weakening after the injection has ceased, the harmonic strength increases significantly during the first 300 turns of storage, before falling off to a constant, lesser strength for the remainder of the storage. The effect is even more pronounced for unchopped beam conditions (Fig. 2), where the harmonic strength increases for a full 1000 turns after the end of injection, and then drops sharply before increasing again until the end of storage. It is not possible to know how long the microbunch structure would have persisted had the unchopped beam been allowed to store for a longer period of time. Regardless, from the standpoint of single microbunch considerations, it is astonishing that the linac microbunch structure amplifies during the beam storage, where energy spread and space-charge effects would have intuited the opposite response.

To investigate the intensity dependence of the microbunch structure during beam storage, we compare singleturn longitudinal profiles for two data sets with different beam intensities but otherwise identical experimental conditions. The two plots shown in Fig. 4 correspond to 

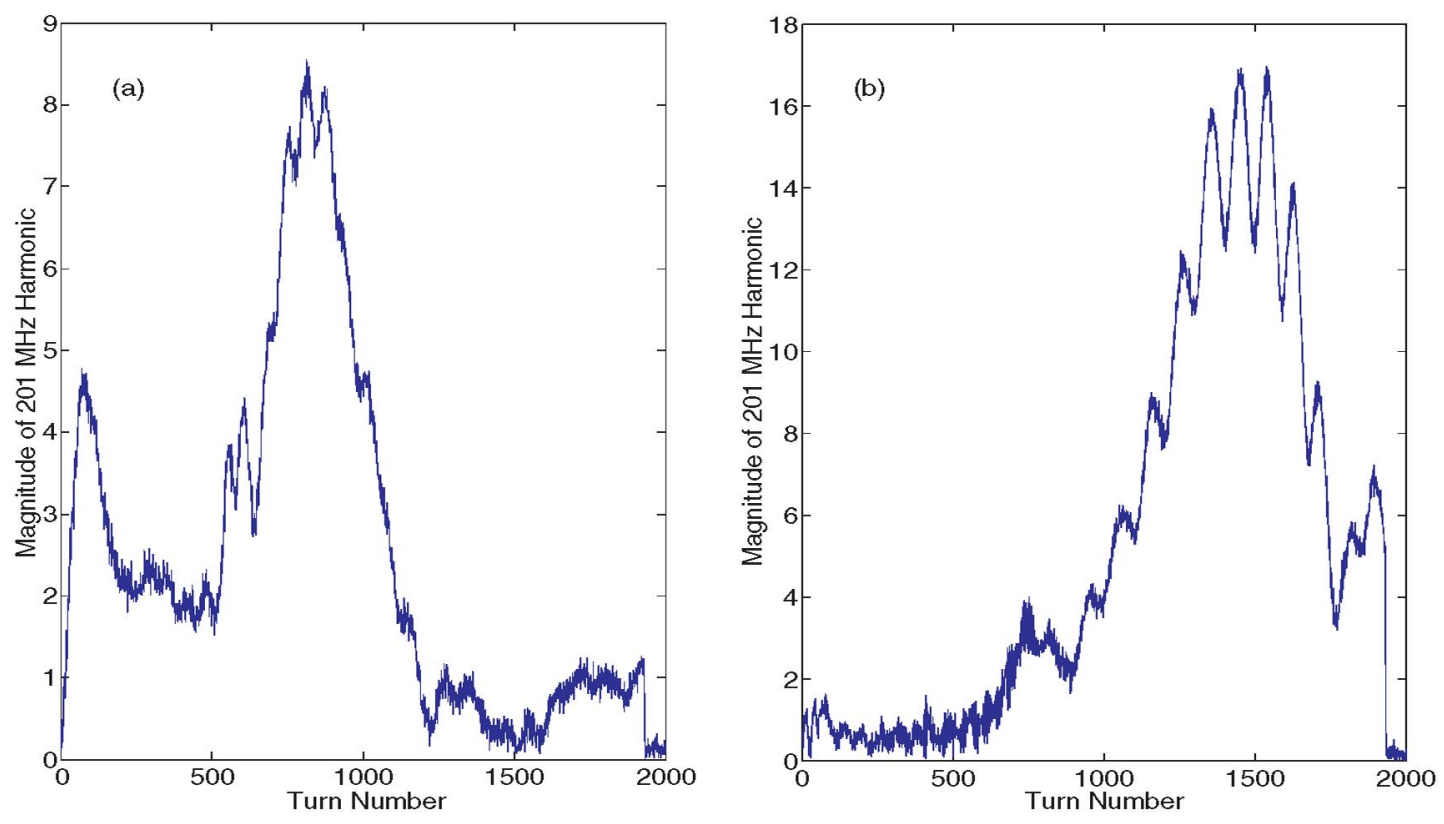

FIG. 3. (Color) (a) Evolution of the 72nd harmonic corresponding to the $201.25 \mathrm{MHz}$ linac microbunch frequency during injection and storage for the chopped beam experiment shown in Fig. 1. (b) The same as (a) but for the unchopped beam experiment shown in Fig. 2 .

longitudinal profiles for a single turn of beam 300 turns after the end injection, for a $70 \mathrm{nC}$ beam and a $210 \mathrm{nC}$ beam. The hash at the top of the profiles is the microbunch signal. Note that it occupies about $5 \%$ of the total beam strength for the $70 \mathrm{nC}$ beam [Fig. 4(a)]. At triple the intensity, i.e., the $210 \mathrm{nC}$ case [Fig. 4(b)], the hash is much

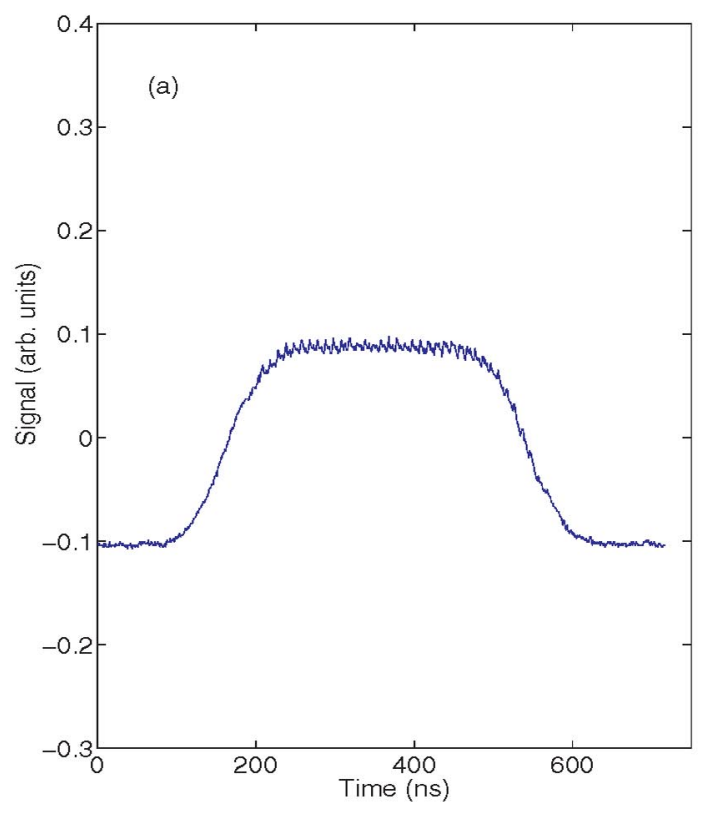

more pronounced, and occupies about $15 \%$ of the beam signal. It is clear that a correlation exists between the intensity of the beam and the strength of the microbunch structure, suggesting that space charge contributes to the sustained microbunch structure of the beam. To investigate the dynamics of the system, and to understand the

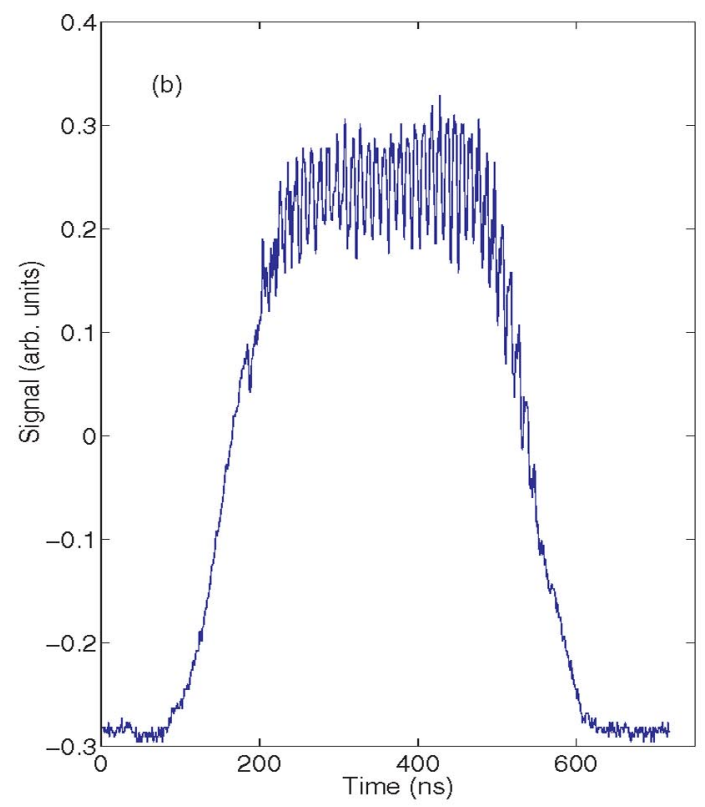

FIG. 4. (Color) (a) Wall current monitor signal for a single turn of a $70 \mathrm{nC}$ chopped beam, 300 turns after the end of injection. The hash at the top is the linac microbunch structure. (b) Wall current monitor signal for a single turn of a $210 \mathrm{nC}$ chopped beam, 300 turns after the end of injection. 
role of space charge in the phenomenon, in the next section we reproduce the chopped beam experiments with detailed particle-in-cell (PIC) tracking simulations.

\section{SIMULATION}

ORBIT is a PIC code developed for realistic simulations of beams in rings and transport lines [11,12]. The code has been successfully benchmarked with experimental data for a number of cases [13,14]. For the simulations presented here, we invoke ORBIT's 1D longitudinal tracking package, which includes an algorithm for modeling the effects of longitudinal space charge and user-defined external impedances [15]. One combined space charge and impedance kick is applied per turn of the beam, adequate for modeling the slow longitudinal phase space evolution of the PSR beam. Although initially the persistent microbunch structure was observed in simulations with the ferrite inductor impedance included, no correlation was found between the sustained structure and the inductor impedance. To demonstrate that space charge is the relevant effect, in the simulations presented here the impedance of the inductive inserts is intentionally omitted. High numerical resolution is attained by slicing the beam longitudinally into 256 bins and using $8 \times 10^{6}$ macroparticles.

(a)

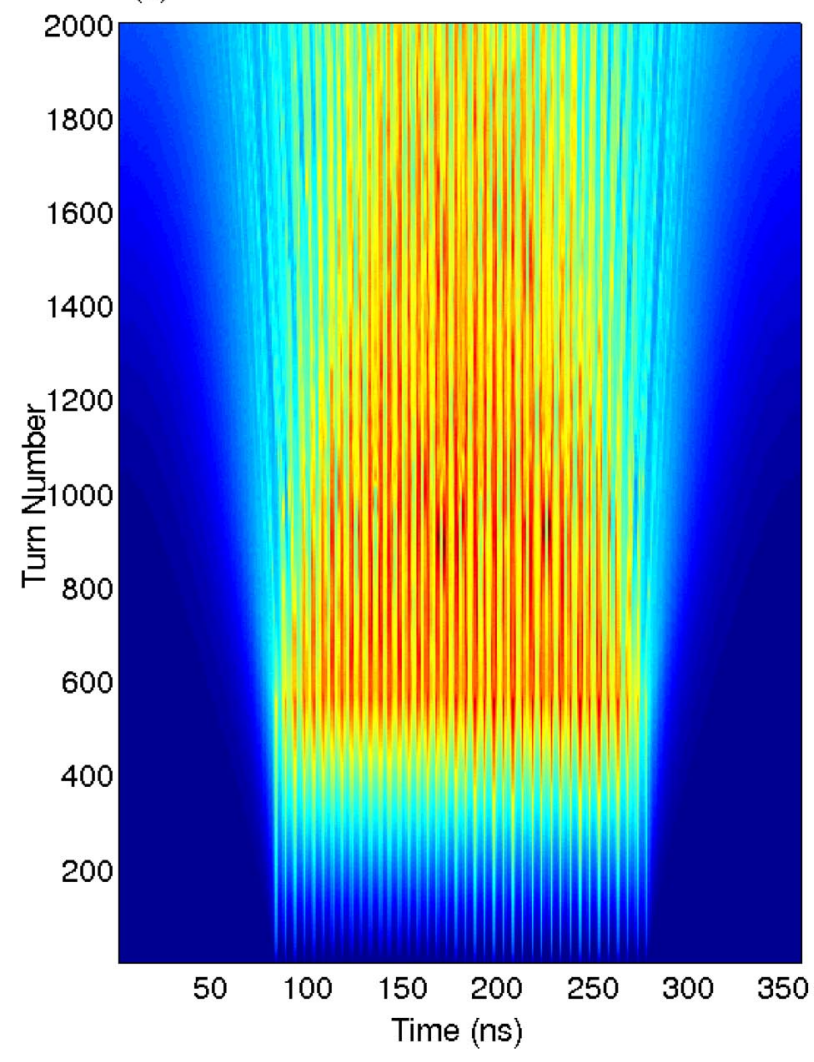

The experimental parameters for the chopped beam case, listed in the previous section, were used in the ORBIT simulations. To allow for direct comparison with the experimental data, turn-by-turn longitudinal beam profiles were plotted with the same resolution as the wall current monitor data $(0.5 \mathrm{~ns})$. The simulations were performed both with and without space charge, and the results are shown in waterfall plot format in Figs. 5(a) and 5(b), respectively. When space charge is included, we observe the same microbunch dynamics as in the experimental data: The $201 \mathrm{MHz}$ structure is locked in place and sustained for at least 800 turns after injection. In the case where no space charge is present, however, the microbunch structure decoheres due to energy spread effects within a few tens of turns after the end of injection.

In a system where energy spread effects are negligible and space charge alone governs the longitudinal dynamics, the steady-state solution for the distribution is always $d \lambda / d \phi=0$, where $\lambda$ is a line density and $\phi$ is the independent variable along the length of the beam. However, in the present system, both space charge and energy spread effects are non-negligible, and evidence from both the experimental and simulated data points to the existence of another steady-state solution.

To understand the dynamics of the system, we investigate the longitudinal phase space of the beam using the

(b)

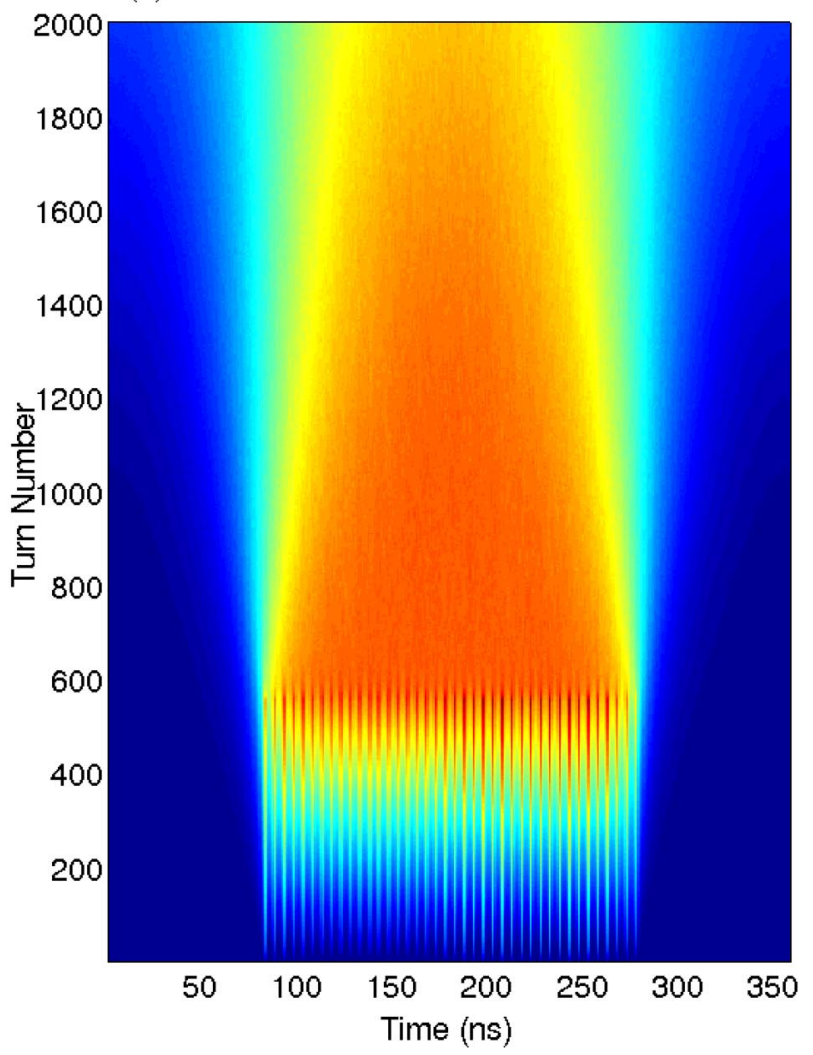

FIG. 5. (Color) (a) Waterfall plot of simulated wall current monitor signal for chopped beam experiments. Space-charge effects are included. (b) Same as in (a) but with no space charge included. 

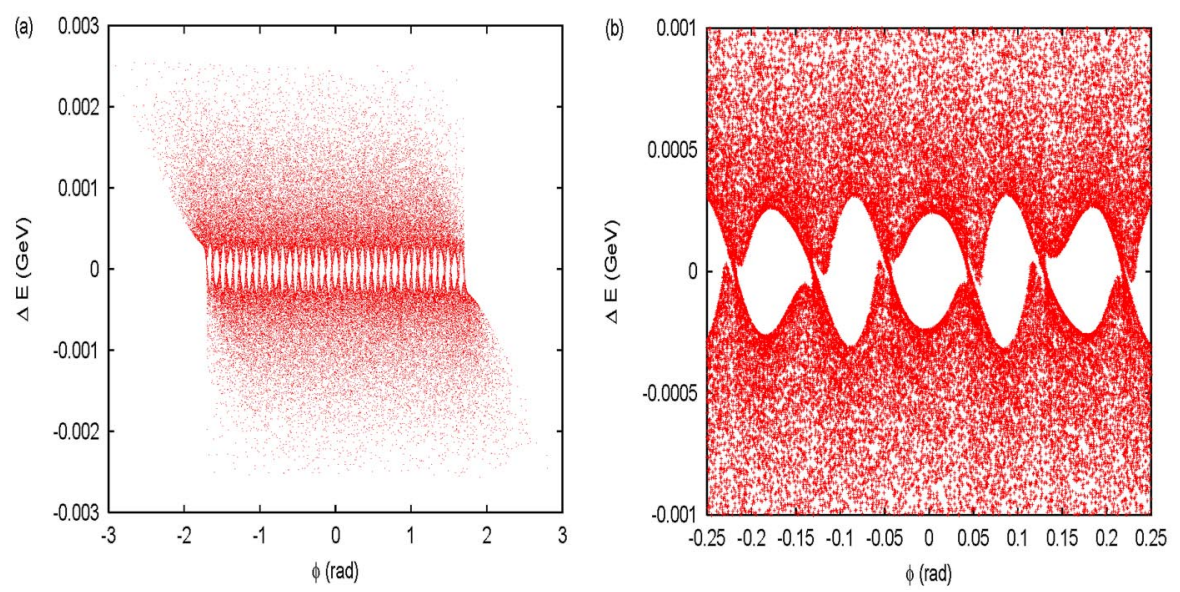

FIG. 6. (Color) (a) Longitudinal phase space of the beam at the end of injection (559 turns) for the chopped beam experiment presented in Fig. 1. (b) Zoom on a smaller portion of the phase space shown in (a).

simulated data. The phase space of the beam at the end of beam injection (turn number 559) is shown in Fig. 6(a). At large energy deviations, the normal phase space drift is observed. At small energy deviations, the linac microbunch structure is visible, where the sharp spikes in density correspond to the locations where the micropulses were accumulated during injection. Figure 6(b) zooms in on the central portion of the phase space, between $-0.25 \leq \phi(\mathrm{rad}) \leq 0.25$ and $-0.001 \leq d E(\mathrm{GeV}) \leq$ 0.001 . Interestingly, a well-defined separatrix is observed in the phase space of the beam. The shape of the separatrix is reminiscent of an external rf bucket, the main differences being that in this system space charge drives the internal $\mathrm{rf}$ voltage, and particles execute unbounded motion in the region outside of the separatrix, rather than executing bounded motion inside of the separatrix. The separatrix forms a series of empty phase space buckets, occurring at exactly the linac microbunch frequency, and giving rise to the $201 \mathrm{MHz}$ density fluctuations observed in the longitudinal wall current monitor signals. Under the influence of these density fluctuations, a particle in this system would move faster through the low density regions and slower through the high density regions. This cycle of fast and slow motion perpetuates a pattern of high density and low density, which in turn reproduces the pattern of fast and slow motion, etc. Intuitively, one can argue that the system should be able to sustain itself in this manner indefinitely. In fact, in the approximation of a system with only two velocity states and two density states, a self-consistent, steady-state analytic solution can easily be found [8]. For more realistic particle density distributions, it is likely that the formation and longevity of these buckets is very sensitive to the experimental conditions.

Looking at the same portion of the phase space $(-0.25 \leq \phi \leq 0.25$ and $-0.001 \leq d E \leq 0.001)$ later in the beam storage, Fig. 7 shows that the buckets are sustained in good form for 250 turns of beam storage [corresponding to Fig. 7(b)], and are just beginning to shear after 650 turns of storage [Fig. 7(c)]. This indicates that the longitudinal phase space was near steady state during the chopped beam experiments. A true steady state could never be achieved for the chopped beam case, since particles will eventually reach the beam gap and exit the varying space-charge potential. An unchopped beam is a more promising candidate for achieving steady state, and will be treated at the end of this section.

To investigate the sensitivities of the dynamics in the chopped beam experiment, we varied the intensity of the beam, the energy spread, and the beam injection rate in the ORBIT simulations. Our results confirm that the longevity of the buckets is sensitive to all of these parameters. A thorough discussion of the dependence on intensity and energy spread for an idealized two-state system can be found in Ref. [8]. For the sake of brevity, here we demonstrate only the sensitivity to the total beam intensity. In this simulation, we reproduce the chopped beam experiment shown in Fig. 1, but with only $100 \mathrm{nC}$ of charge, and all other parameters unaltered. Figure 8 shows the results. Again, the formation of buckets is observed at the end of injection, but the buckets shear much more rapidly than in the $210 \mathrm{nC}$ case (Fig. 7), indicating that the system is farther from steady state. The shearing of the buckets for the lower intensity case translates to a weaker microbunch structure in the wall current monitor signal, similar to what was observed in Fig. 3. At some low intensity, the separatrix should disappear entirely.

In addition to the ORBIT simulations, we have developed an independent calculation which numerically solves the Vlasov equation for a single microbunch with periodic boundary conditions in the longitudinal coordinate. These periodic boundary conditions are appropriate to simulate the unchopped beam experiments. For the $720 \mathrm{nC}$ unchopped experiment shown in Fig. 2, we find 

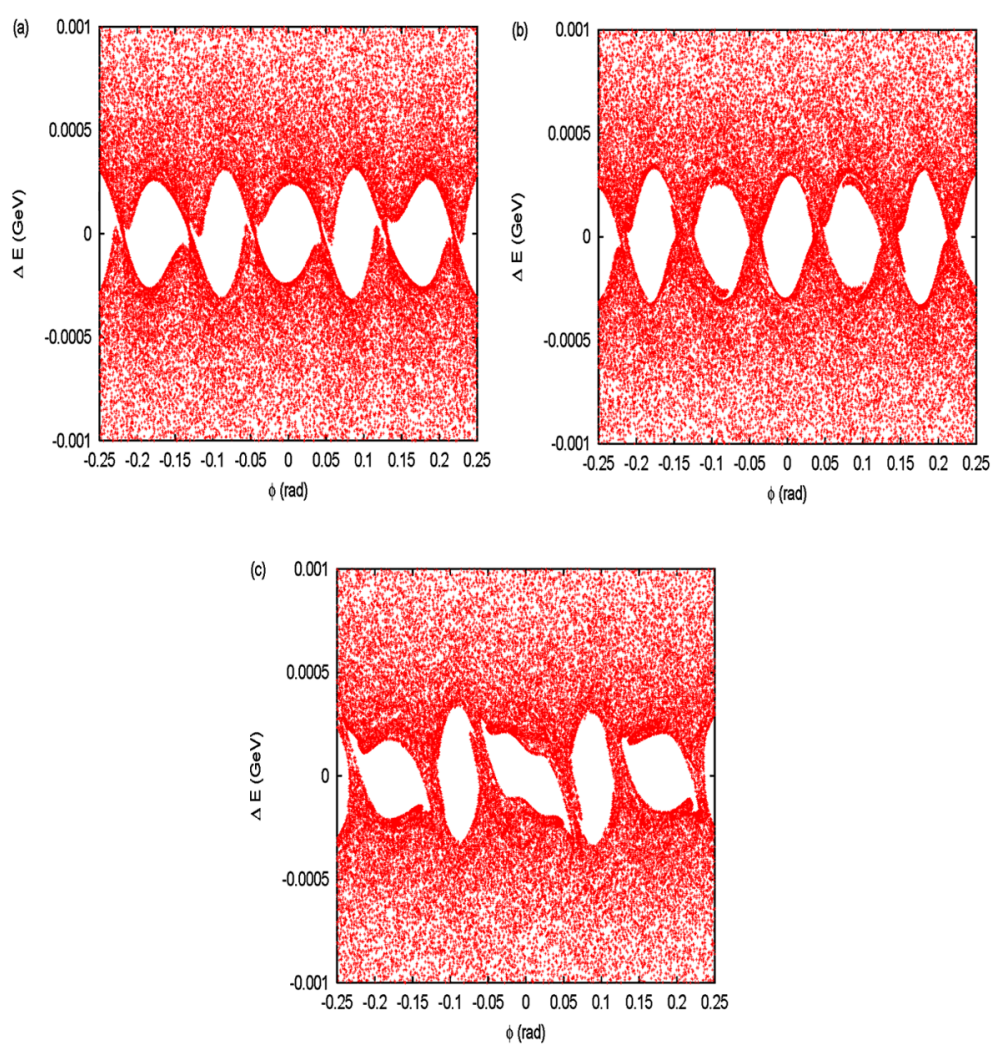

FIG. 7. (Color) (a) Longitudinal phase space of the beam at the end of injection (559 turns) for the chopped beam experiment [same as Fig. 6(b)]. (b) Phase space after 250 turns of storage. (c) Phase space after 650 turns of storage.
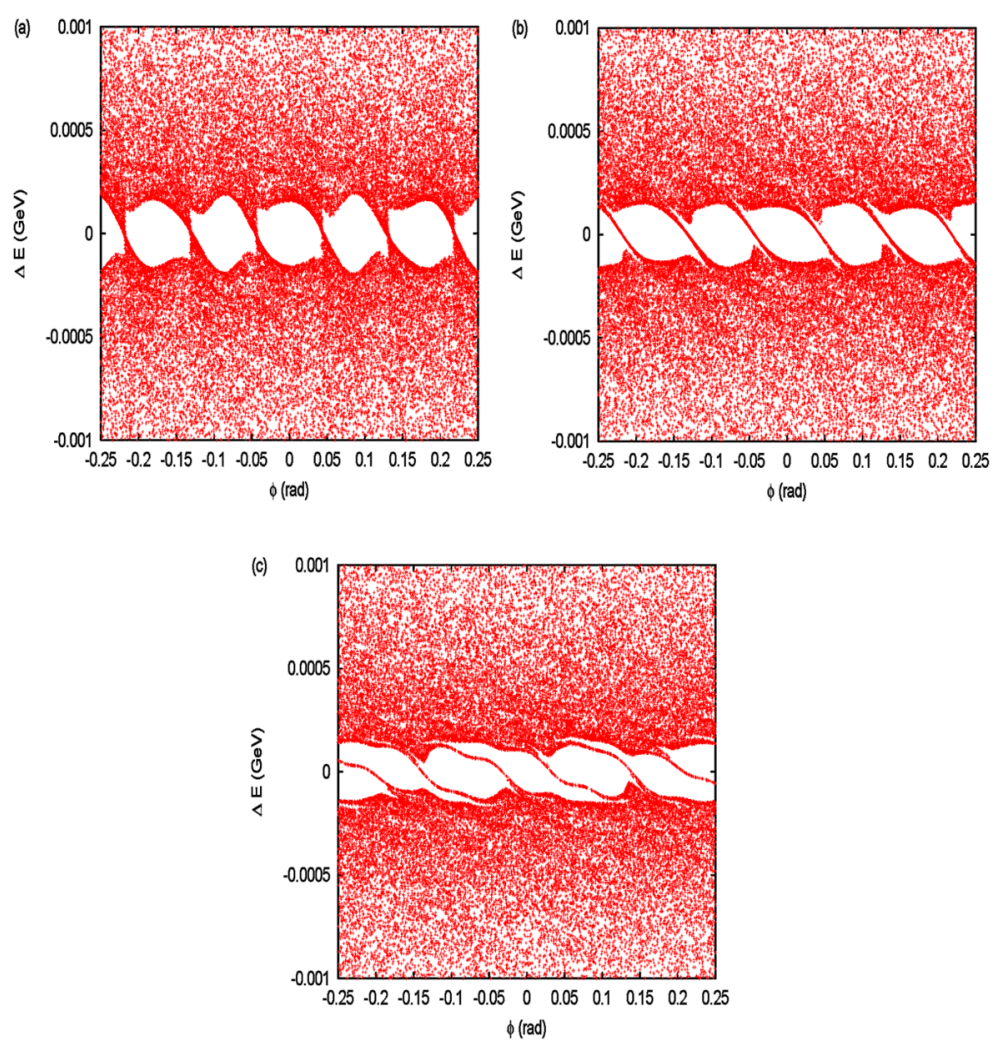

FIG. 8. (Color) (a) Longitudinal phase space of the beam at the end of injection for a $100 \mathrm{nC}$ chopped beam. (b) Phase space after 250 turns of storage. (c) Phase space after 650 turns of storage. 

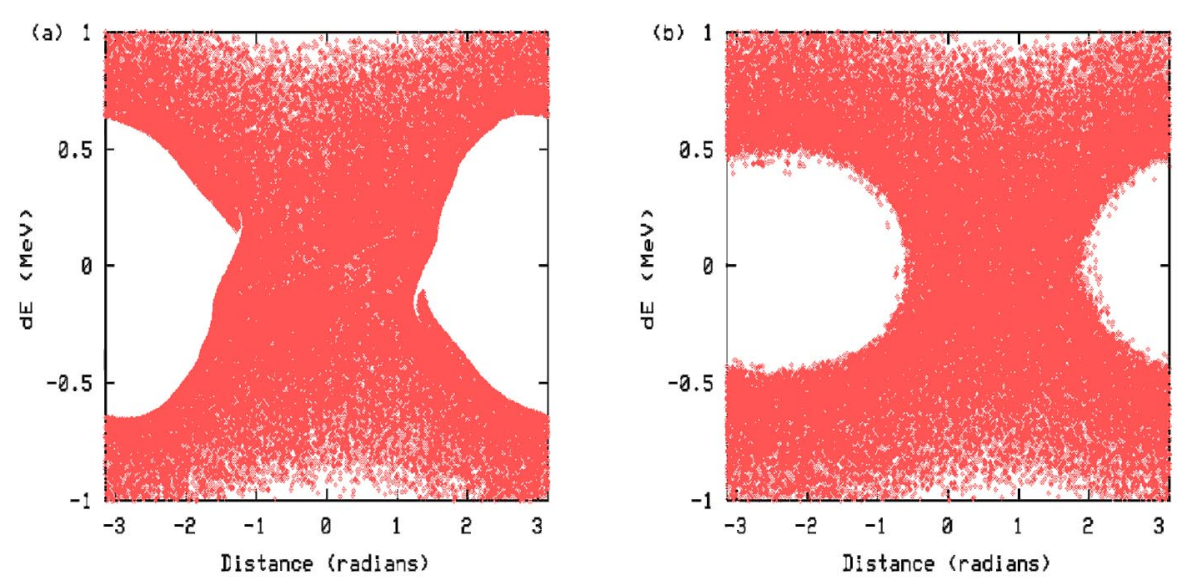

FIG. 9. (Color) (a) Longitudinal phase space of a single microbunch at the end of injection for a $720 \mathrm{nC}$ unchopped beam. (b) Longitudinal phase space of a single microbunch after 10000 turns of storage for a $720 \mathrm{nC}$ unchopped beam.

that the microbunch structure is sustained for up to 10000 turns after the end of injection; we did not simulate beyond this limit. Figure 9 shows the phase space for the single microbunch at the end of injection, and after 10000 turns of storage. Additionally, the Vlasov calculations show the same sensitivity to beam intensity and energy spread as the ORBIT simulations.

\section{ANALYTIC STEADY-STATE SOLUTIONS}

In this section we demonstrate analytically the existence of steady-state longitudinal density perturbations under the influence of internal, defocusing space-charge forces. Specifically, we derive self-consistent, steadystate solutions for a Hamiltonian system with a periodic space-charge potential; no external impedances are included in our analysis.

First, consider the longitudinal Hamiltonian with space charge,

$$
\begin{gathered}
H=\frac{1}{2} h \varepsilon^{2}+k \Lambda(\phi), \\
h=\frac{|\eta| 2 \pi P}{\beta^{2} \varepsilon_{o}}, \\
k=\frac{4 \pi P}{\gamma^{3} L} r_{o} N \varepsilon_{o}\left[\ln \left(\frac{b}{a}\right)+\frac{1}{2}\right],
\end{gathered}
$$

where $\Lambda$ is the particle density distribution, $\eta=\frac{1}{\gamma_{T}^{2}}-\frac{1}{\gamma^{2}}$, $\beta$ and $\gamma$ are the usual relativistic factors, $P$ is the number of periods, $L$ is the length of the ring, $\varepsilon_{o}=\gamma m c^{2}, \varepsilon \rightarrow$ $\varepsilon-\varepsilon_{o}$ is the energy deviation, $r_{o}$ is the classical proton radius, $N$ is the number of particles, $\frac{b}{a}$ is the ratio of the beam pipe radius to the beam radius, $\phi$ is the longitudinal coordinate with $\phi \equiv 2 \pi \frac{P}{L} z$, and $z$ is the independent variable along the length of the ring. We define $\Lambda$ as an arbitrary, periodic particle line density, constrained to $\Lambda>0$ and normalized as follows:

$$
\begin{gathered}
\Lambda(\phi)=1+\sum_{n=1}^{\infty} \Lambda_{n} \cos (n \phi), \\
\int_{-\pi}^{\pi} \Lambda d \phi=2 \pi .
\end{gathered}
$$

Let us introduce the particle phase space distribution function $f(\phi, \varepsilon)$. Then self-consistency demands that

$$
\Lambda(\phi)=\int_{-\infty}^{\infty} f(\phi, \varepsilon) d \varepsilon
$$

We enforce periodicity over the range $-\pi \leq \phi \leq \pi$ in $H$ and $f$. Steady state requires $\frac{d f}{d t}=0$, which is guaranteed when the distribution function is a function of the Hamiltonian, i.e., $f=f(H)$. By symmetry arguments, from the self-consistency equation [Eq. (6)], we have

$$
\int_{0}^{\infty} f(H) d \varepsilon=\frac{\Lambda}{2}
$$

which gives

$$
\int_{k \Lambda}^{\infty} \frac{d H f(H)}{\sqrt{H-k \Lambda}}=\frac{1}{k} \sqrt{\frac{h}{2}}(k \Lambda) .
$$

We now define the function $g(H)=\frac{c k}{\sqrt{h / 2}} f(H)$ and note that it is sufficient to solve for $g(H)$ in the similar system

$$
\int_{x}^{\infty} \frac{d H g(H)}{\sqrt{H-x}}=c x
$$

where $x=k \Lambda$ and $c$ is a constant. Assuming that $g(H)=$ 0 for $H>H_{\max }$ and scaling such that $\eta=H / H_{\max }, x \rightarrow$ $x / H_{\max }$, and $c \rightarrow c / \sqrt{H_{\max }}$, we get

$$
\int_{x}^{1} \frac{d \eta g(\eta)}{\sqrt{\eta-x}}=c x
$$




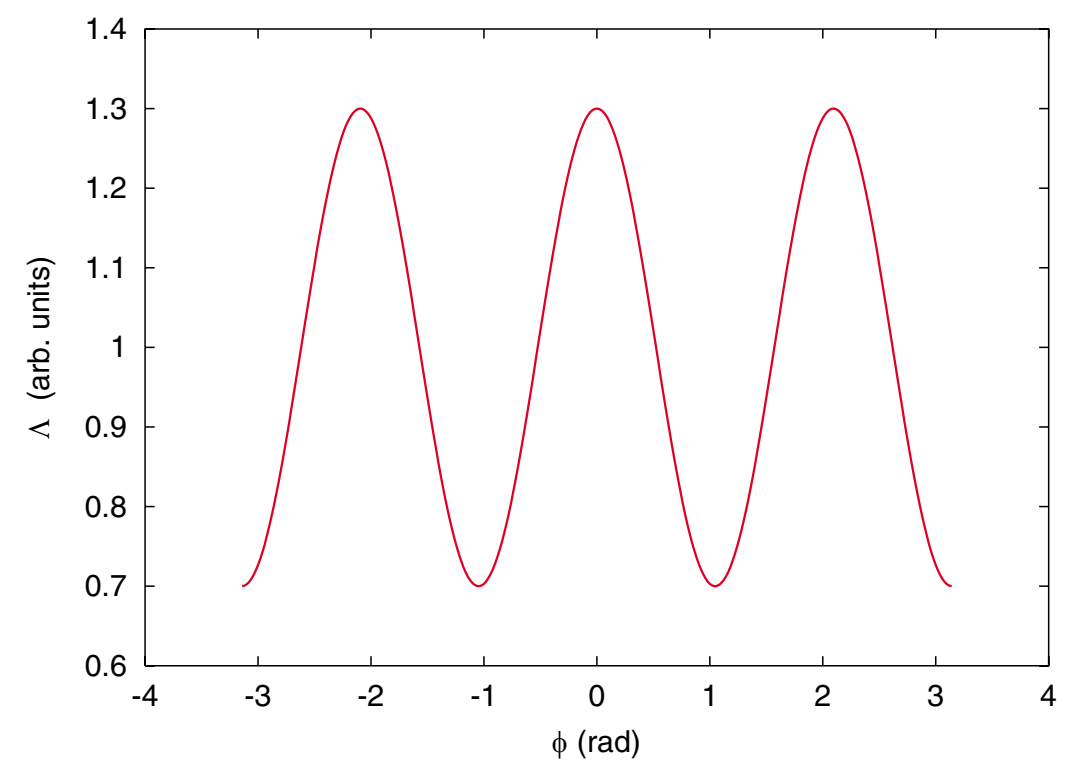

FIG. 10. (Color) The line density function used in the steady-state Hamiltonian formulation.

A solution for the above equation is given by

$$
g(\eta)=\frac{c}{\pi}\left[\frac{1}{\sqrt{1-\eta}}-2 \sqrt{1-\eta}\right]
$$

Requiring that the distribution function be positive, i.e., $g(\eta)>0$, physical solutions exist only when

$$
x=\frac{k \Lambda}{H_{\max }} \geq \frac{1}{2} .
$$

Substituting back, the self-consistent, steady-state solution for $f(H)$ is given by

$$
f(H)=\frac{1}{k \pi} \sqrt{\frac{h}{2}} \sqrt{H_{\max }}\left[\frac{1}{\sqrt{1-\left(H / H_{\max }\right)}}-2 \sqrt{1-\frac{H}{H_{\max }}}\right]
$$

when $H_{\max } / 2<H<H_{\max } ; f(H)=0$ otherwise.

For the line density function given in Eq. (4), the constraint (12) implies a condition on $\Lambda$ given by

$$
\frac{H_{\max }}{2 k}<1+\sum_{n=1}^{\infty} \Lambda_{n} \cos (n \phi)<\frac{H_{\max }}{k} .
$$

Therefore, a steady state exists for any periodic beam potential described by Eq. (4), as long as the constraint Eq. (14) is met, which corresponds to a relatively narrow band of Hamiltonian values. Here we do not guarantee stability of the solutions, and instead leave this analysis for later work.

As an example, we take the case of a single-harmonic sinusoidal beam potential with harmonic number $n=3$ (a)

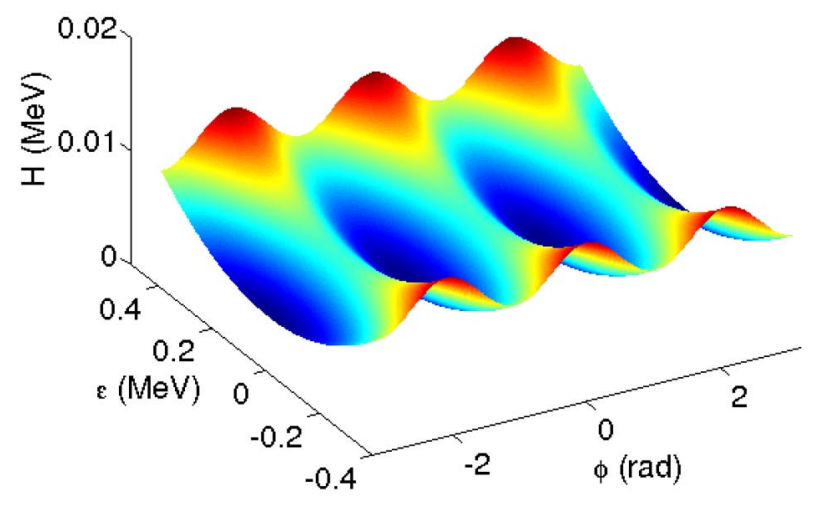

(b)

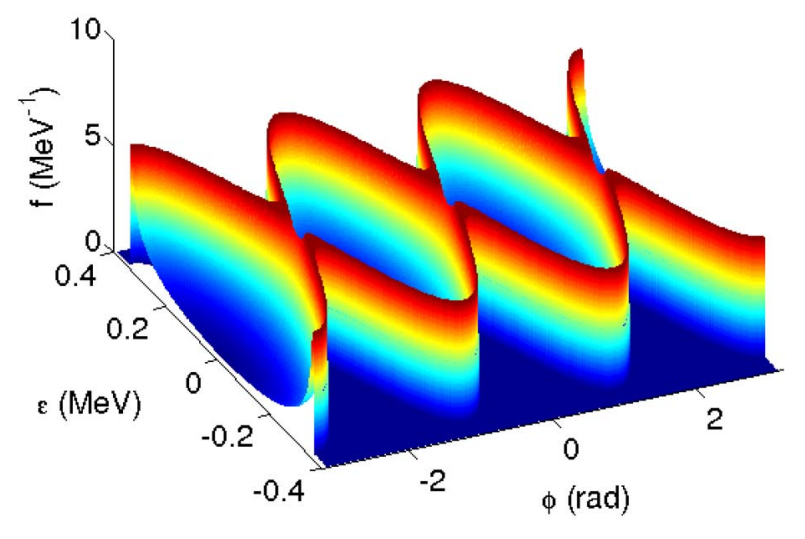

FIG. 11. (Color) (a) The steady-state Hamiltonian surface for the line density specified in Eq. (15). (b) The associated steadystate distribution function. In both plots, the blue color indicates the lowest values and the red color indicates the highest values. 
given by

$$
\Lambda=1+0.3 \cos (3 \phi),
$$

with $1000 \mathrm{nC}$ of charge, and other parameters taken for the PSR beam. The particle line density is plotted in Fig. 10. Figure 11(a) shows the surface of the resulting steady-state Hamiltonian, with clearly defined saddle points, and wells located at the minimum of the spacecharge potential. The associated steady-state distribution function is shown in Fig. 11(b). The plot has been truncated at the $98 \%$ value in order to avoid the singularity in $f(H)$ that occurs when $H=H_{\max }$. We see that the steadystate distribution function peaks outside of the separatrix, where the potential is large, and very few particles occupy the potential wells inside the separatrix. This deficit of particles near the minimum of the space-charge potential is in good agreement with what we observe in the PSR beam.

\section{CONCLUSIONS}

We have examined experimentally observed, longlived microbunch structure in the PSR machine. Simulations of the experiment indicate that a separatrix is formed in the longitudinal phase space of the beam between the linac microbunches. Because of the initial injection conditions in the PSR, all particles are confined to the outside of the separatrix, thus forming a series of empty buckets in the phase space which give rise to the sustained microbunch structure. The PSR beam is found to be near the steady-state condition during the experiments, such that the separatrix is maintained for a long period of time.

The separatrix formation in the phase space results from the interaction of space charge and energy spread effects, and occurs for a certain balance of experimental conditions. In this system, the maximum particle density occurs near the maximum of the space-charge potential. Using Hamiltonian formalism and considering only space charge and energy spread effects, we have derived self-consistent steady-state solutions for a set of periodic space-charge potentials. The resulting steady state is confined to a relatively narrow band in the Hamiltonian space. Experimentally, however, a beam needs only to be somewhere near the steady state to exhibit long-lived phase space structure. Such conditions should be met easily in high intensity synchrotrons, where typical storage times are on the order of a few milliseconds and thus a near steady state would result in sustained structure throughout the entire storage.

\section{ACKNOWLEDGMENTS}

The authors would like to thank John Galambos and Mike Plum for useful discussions, and Sasha Aleksandrov for assistance with MATLAB. This work is supported by SNS through UT-Battelle, LLC, under Contract No. DE-AC05-00OR22725 for the U.S. DOE, and by Los Alamos National Laboratory under Contract No. W-7405-ENG-36. The Los Alamos National Laboratory is operated by the University of California for the U.S. DOE. The SNS is a partnership of six national laboratories: Argonne, Brookhaven, Jefferson, Lawrence Berkeley, Los Alamos, and Oak Ridge.

[1] H. Schamel, Phys. Rev. Lett. 79, 2811 (1997).

[2] H. Schamel, in Proceedings of the 2000 European Particle Accelerator Conference, Vienna, 2000 (CERN, Geneva, 2000).

[3] M. Blaskiewicz, J. Wei, A. Luque, and H. Schamel, Phys. Rev. ST Accel. Beams 7, 044402 (2004).

[4] R. Baartman, in Proceedings of the 1991 Particle Accelerator Conference, San Francisco, 1991 (IEEE, Piscataway, NJ, 1991), p. 1731.

[5] M. D'Yachkov and R. Baartman, in Proceedings of the High Energy Accelerator Conference (HEAC92), Hamburg, 1992 (to be published), p. 1064.

[6] S. I. Tzenov and P. L. Colestock, Fermilab Technote No. Fermilab-Pub-98-258, 1998.

[7] L. K. Spentzouris, J. F. Ostiguy, and P. L. Colestock, Phys. Rev. Lett. 76, 620 (1996).

[8] S. Koscielniak, S. Hancock, and M. Lindroos, Phys. Rev. ST Accel. Beams 4, 044201 (2001).

[9] C. Beltran, Ph.D. thesis, Indiana University, 2004 (unpublished).

[10] S. Cousineau, C. Beltran, J. Holmes, and R. Macek, in Proceedings of the 2004 European Particle Accelerator Conference, Lucerne, 2004 (CERN, Geneva, 2004).

[11] J. Galambos, J. Holmes, A. Luccio, D. Olsen, and J. Beebe-Wang, ORBIT User's Manual, http:// www.sns.gov//APGroup/Codes/Codes.htm.

[12] J. Holmes, S. Cousineau, J. Galambos, A. Shishlo, Y. Sato, W. Chou, M. Michelotti, and F. Ostiguy, ICFA Beam Dynamics Newsletter 30, 100 (2003).

[13] J. Galambos, S. Danilov, J. Holmes, D. Jeon, F. Neri, D. K. Olsen, and M. Plum, Phys. Rev. ST Accel. Beams 3, 034201 (2000).

[14] S. Cousineau, J. Holmes, J. Galambos, A. Fedotov, J. Wei, and R. Macek, Phys. Rev. ST Accel. Beams 6, 074202 (2003).

[15] J. MacLachlan, Fermilab Technical Note No. FN-446. 